\title{
Genetic variation in PEAR1, cardiovascular outcomes and effects of aspirin in a healthy elderly population
}

Joshua P. Lewis ${ }^{1 \#}$, Moeen Riaz ${ }^{2 \#}$, Sophia $\mathrm{Xie}^{2}$, Galina Polekhina ${ }^{1}$, Rory Wolfe ${ }^{2}$, Mark Nelson ${ }^{2,3}$, Andrew M Tonkin ${ }^{2}$, Christopher M Reid ${ }^{2,4}$, Anne M Murray ${ }^{5}$, John J McNeil ${ }^{2}$, Alan R Shuldiner ${ }^{1 \uparrow} \&$ Paul Lacaze ${ }^{2 \pi^{*}}$.

\# Joint first authors $\llbracket$ Joint senior authors

${ }^{1}$ Department of Medicine, Program for Personalized and Genomic Medicine, University of Maryland School of Medicine, Baltimore, MD 21201

${ }^{2}$ Department of Epidemiology and Preventive Medicine, School of Public Health and Preventive Medicine, Monash University, Melbourne, VIC, Australia.

${ }^{3}$ Menzies Institute for Medical Research, University of Tasmania, Hobart, TAS, Australia

${ }^{4}$ School of Public Health, Curtin University, Perth, WA, Australia

${ }^{5}$ Berman Center for Outcomes and Clinical Research, Hennepin Healthcare Research Institute, Hennepin Healthcare, Minneapolis, MN, USA

*Corresponding author: paul.lacaze@monash.edu, +61 (0)409 416 931. School of Public Health and Preventive Medicine, Monash University, Level 5 The Alfred Centre, VIC 3004, Australia.

Conflict of interest statement: J.P.L. receives grant funding to study the pharmacogenomics of antiplatelet agents. A.R.S. is an employee of Regeneron Pharmaceuticals and receives compensation for his employment. All other authors declared no competing interests for this work.

Funding information: The ASPREE study was supported by grants from the National Institute on Aging and the National Cancer Institute at the National Institutes of Health (U01AG029824 and R01HL137922), and by grants from the National Health and Medical Research Council of Australia (334047 and 1127060), by Monash University and the Victorian Cancer Agency, and by Bayer AG for This article has been accepted for publication and undergone full peer review but has not been through the copyediting, typesetting, pagination and proofreading process, which may lead to differences between this version and the Version of Record. Please cite this article as doi: $\underline{10.1002 / C P T .1959}$

This article is protected by copyright. All rights reserved 
the provision of aspirin and placebo. P.L is supported by a National Heart Foundation Future Leader Fellowship and J.P.L is supported by R01HL137922 from National Institutes of Health.

Keywords: Genetics, aspirin, platelet aggregation, cardiovascular disease.

This article is protected by copyright. All rights reserved 


\section{ABSTRACT}

The platelet endothelial aggregation receptor-1 (PEARI) rs12041331 variant has been identified as a genetic determinant of platelet aggregation in response to antiplatelet therapies, including aspirin. However, association with atherothrombotic cardiovascular events is less clear, with limited evidence from large trials. Here, we tested association of rs12041331 with cardiovascular events and aspirin use in a randomized trial population of healthy older individuals. We undertook post-hoc analysis of $\mathrm{N}=13,547$ participants of the ASPirin in Reducing Events in the Elderly (ASPREE) trial, median age 74 years. Participants had no previous diagnosis of atherothrombotic cardiovascular disease at enrolment, and were randomized to either $100 \mathrm{mg}$ daily low-dose aspirin or placebo for median 4.7 years follow-up. We used Cox proportional hazard regression to model the relationship between rs12041331 and the ASPREE primary cardiovascular disease endpoint (CVD), and composites of major adverse cardiovascular events (MACE) and ischaemic stroke (STROKE); and bleeding events; major hemorrhage (MHEM) and intracranial bleeding (ICB). We performed whole-population analysis using additive and dominant inheritance models, then stratified by treatment group. Interaction effects between genotypes and treatment group were examined. We observed no statistically significant association $(\mathrm{P}<0.05)$ in the population, or by treatment group, between rs12041331 and cardiovascular or bleeding events in either model. We also found no significant interaction effects between rs12041331-A and treatment group, for CVD ( $\mathrm{P}=0.65)$, MACE $(\mathrm{P}=0.32)$, STROKE $(\mathrm{P}=0.56)$, MHEM $(\mathrm{P}=0.59)$ or ICB $(\mathrm{P}=0.56)$. The genetic variant PEAR1 rs12041331 is not associated with cardiovascular events in response to low-dose aspirin in a healthy elderly population.

This article is protected by copyright. All rights reserved 


\section{INTRODUCTION}

2 Interindividual variability in response to antiplatelet medications including aspirin can result in 3 inadequate platelet inhibition and subsequent cardiovascular and cerebrovascular events. Clinical and 4 anthropometric factors are known to contribute to variable aspirin efficacy including age, gender, body mass index (BMI), diabetes mellitus (DM), compliance, and certain drug-drug interactions(1-3). In addition, heritability estimates, as assessed by ex vivo agonist-stimulated platelet aggregation, suggest that a large proportion of variation in response to aspirin may be attributable to genetic factors(4). Indeed, multiple pharmacogenetic investigations ranging from candidate gene reports to genome-wide association 9 studies have been conducted to identify novel genetic determinants of aspirin response. Identification of 10 genetic polymorphisms that lead to suboptimal platelet control may have important implications in

11 primary and secondary prevention of adverse clinical events in patients with an indication for this 12 medication.

13 The platelet endothelial aggregation receptor 1 (PEAR1) gene encodes a type 1 membrane protein, that is 14 highly expressed in platelets and endothelial cells(5) and critical in haematological processes including 15 megakaryopoiesis, thrombopoiesis, and $\alpha \operatorname{IIb} \beta 3$-mediated platelet aggregation $(6,7)$. Genetic variants in 16 the PEAR1 gene have been associated with platelet aggregation $(8,9)$ and in response to antiplatelet agents 17 including aspirin(10-12), clopidogrel(13), ticagrelor(14), and prasugrel(15). Most notably, an intronic 18 variant rs12041331 in the PEARl gene is among the strongest genetic modifiers of platelet aggregation, 19 particularly in those treated with aspirin, and results in allele-specific differences in $\mathrm{H} 3 \mathrm{~K} 4 \mathrm{Me} 1$ 20 methylation leading to differential gene and protein expression(11, 16, 17). Despite the evidence demonstrating the influence of PEARI rs12041331 on agonist-stimulated platelet aggregation in aspirintreated patients, the impact of this variant on thrombotic and bleeding events remains unclear.

23 In this investigation, we assessed the interaction between PEAR1 rs12041331 and randomized aspirin use 24 in 13,547 participants of the Aspirin in Reducing Events in the Elderly (ASPREE) trial. Specifically, 25 individuals with no previous diagnosis of atherothrombotic cardiovascular disease were randomized to 26 either $100 \mathrm{mg}$ daily low-dose aspirin or placebo for median 4.7 years and relationships between 27 rs12041331 genotypes and cardiovascular events, as well as clinically significant bleeding events, were 28 examined. Moreover, gene $\mathrm{x}$ environment (i.e. aspirin treatment) interaction models were used to test 29 whether the effect of rs12041331 genotypes on clinical endpoints varied based on aspirin use.

This article is protected by copyright. All rights reserved 


\section{METHODS}

\section{Study population}

32 Details of the ASPREE trial are described previously(18, 19). Briefly, ASPREE was a double-blind, 33 randomized (1:1), placebo-controlled trial to examine the effect of daily low-dose aspirin on disability34 free survival in healthy older individuals(19-21). At enrolment, the 19,114 participants of the ASPREE 35 trial had no current symptoms or prior history of cardiovascular events, including: myocardial infarction, 36 heart failure, stroke, transient ischemic attack, atrial fibrillation or high blood pressure. Participants also 37 had no dementia diagnosis, physical disability, or illness likely to cause death within 5 years at enrolment. 38 Participants were followed prospectively, monitored for a range of primary and secondary outcomes, 39 including cardiovascular events, dementia, persistent disability and cancer. Participants were enrolled 40 from March 2010 through December 2014, with follow-up data collected until June 2017 (median follow41 up of 4.7 years). All investigational protocols were approved by local Institutional Review Boards across 42 Australia and the United States and conformed to the principles outlined in the Declaration of Helsinki. 43 Each participant gave informed consent prior to enrolment.

\section{Outcomes}

45 We analysed the relationship between aspirin use and the common variant PEAR1 rs12041331, in relation 46 to clinically adjudicated cardiovascular and bleeding events in ASPREE. Endpoints analysed included the 47 primary cardiovascular disease endpoint in the ASPREE study (CVD), and composites of major 48 cardiovascular events considered most likely to be impacted by aspirin; including major adverse 49 cardiovascular events (MACE) and ischaemic stroke (STROKE); and clinically significant bleeding 50 events, including major hemorrhage (MHEM) and intracranial bleeding (ICB). Details of ASPREE 51 endpoints are described previously(21).

\section{Genetic analysis}

53 Ethical approval for genetic analysis was obtained from the Alfred Hospital Human Research Ethics 54 Committee (390/15). Genome-wide SNP genotyping of $\mathrm{N}=14,177$ samples collected by the ASPREE 55 Healthy Ageing Biobank was performed using the Axiom 2.0 Precision Medicine Diversity Research 56 Array (Thermo Fisher Scientific, CA, USA) following standard protocols, at the Ramaciotti Centre for 57 Genomics, University of New South Wales, Australia. Samples were excluded based on poor quality control (QC) metrics or genotyping performance $(\mathrm{N}=426)$ and other filters including gender discordance $(\mathrm{N}=80)$, and relatedness $(\mathrm{N}=124)$. In the final dataset, a total of $\mathrm{N}=13,547$ samples from individuals of predominantly Non-Finish European ancestry were available for genetic analyses. Individual variants 
were excluded based on genotyping rate $(<95 \%)$ and Hardy-Weinberg equilibrium. Imputation was performed on European-ancestry samples using the haplotype reference consortium (HRC) panel on the University of Michigan imputation server(22). Post-imputation QC removed all variants with $<0.3$ imputation quality scores. The imputation quality score of the variant rs 12041331 was $\mathrm{R}^{2}=0.99$.

\section{Statistical analysis}

We use Cox proportional hazards regression models to analyze the relationship between rs12041331-A allele and cardiovascular events, adjusting for variables related to cardiovascular risk, including: age, gender, smoking status, alcohol intake, high density lipoproteins (HDL), low density lipoprotein (LDL)cholesterol, triglycerides (TG), total cholesterol (TC), hypertension, body mass index (BMI), diabetes mellitus (DM) and statin use prior to enrolment. Analysis of the entire cohort $(\mathrm{N}=13,547)$ was performed using an additive model of inheritance (GG vs AG vs AA) and dominant model (GG vs AG/AA), followed by stratified analysis of the placebo $(\mathrm{N}=6,806)$ and aspirin $(\mathrm{N}=6,741)$ groups, separately. Then the modifying effect of the rs12041331-A genotype on association between incident CVD events and aspirin treatment was analysed using an interaction term in a multivariable Cox regression model using participants from both groups, under both the additive and dominant genetic models. We calculated the minimal detectable effect for all endpoints under both models. All analyses were performed with Stata 15.1 (Stata Corp., Tex., USA) and R (R Core Team, 2014). P-values $<0.05$ were considered as statistically significant (two-sided). Figures were produced using the package ggplot2. 


\section{RESULTS}

The 13,547 genotyped participants had a median age of 73.9 years and were $54.7 \%$ female. The distributions of age, sex, plasma lipids and BMI variables were balanced between the groups (Table 1). Genotype distribution for rs12041331 was also balanced between the placebo and aspirin groups, with GA heterozygotes found at $16.3 \%(\mathrm{~N}=1,112)$ and $15.3 \%(\mathrm{~N}=1,034)$ respectively, and AA homozygotes at $0.9 \%(\mathrm{~N}=64)$ and $0.9 \%(\mathrm{~N}=60)$ respectively (Figure 1 , Table 2$)$.

We did not observe evidence of association between PEAR1 rs12041331 and cardiovascular or bleeding events when stratifying the cohort by treatment effect (Table 3), based on using an additive (Figure 2) or dominant (Figure 3) models of inheritance. In the placebo group, when comparing major allele homozygotes to heterozygotes or minor allele homozygotes using the additive model, no difference in event rate was noted for $\mathrm{CVD}(\mathrm{P}=0.27$ and $\mathrm{P}=0.62$, respectively), MACE $(\mathrm{P}=0.66$ and $\mathrm{P}=0.73$, respectively), STROKE ( $\mathrm{P}=0.92$ and $\mathrm{P}=0.95$, respectively), MHEM ( $\mathrm{P}=0.57$ and $\mathrm{P}=0.61$, respectively) or $\operatorname{ICB}(\mathrm{P}=0.92$ and $\mathrm{P}=0.95$, respectively). Hazard ratio [HR] point estimates (Table 3 ) were generally in the same direction as observed when evaluating the entire cohort (see below) (Table S1).

Similarly, there was no evidence to suggest that PEAR1 rs12041331 genotype impacted cardiovascular or bleeding event rate in participants treated with aspirin (Table 3). In individuals randomized to aspirin, rs12041331 A-allele carrier status did not result in statistically significant differences in atherothrombotic event rates (CVD, MACE and STROKE) or bleeding events (MHEM and ICB) regardless if individuals carried 1 (CVD $\mathrm{P}=0.14$, MACE $\mathrm{P}=0.11$, STROKE $\mathrm{P}=0.51$, MHEM $\mathrm{P}=0.92$, and $\mathrm{ICB} \mathrm{P}=0.51$ ) or 2 (CVD $\mathrm{P}=0.78$, MACE $\mathrm{P}=0.85$, STROKE $\mathrm{P}=0.76$, MHEM $\mathrm{P}=\mathrm{N} / \mathrm{A}$, and ICB $\mathrm{P}=0.76$ ) copies of the A-allele (Table 3). We repeated these analyses using a dominant model of inheritance (wild-type GG vs all AA/AG carriers) and also found no significant associations (Table S2).

In the combined cohort (both aspirin- and placebo-treated groups), no association was observed between rs12041331 A-allele carrier status and atherothrombotic events for either the additive (Table S1) or dominant (Table S2) genetic models. Specifically, no statistical difference in event rates for CVD, MACE and STROKE were seen when comparing PEAR1 rs12041331 major allele homozygotes to either heterozygotes $(\mathrm{HR}=0.81 ; 95 \%$ confidence interval $[\mathrm{CI}] 0.64$ to $1.02 ; \mathrm{P}=0.07, \mathrm{HR}=0.84,95 \% \mathrm{CI} 0.64$ to $1.09, \mathrm{P}=0.19$, and $\mathrm{HR}=0.94,95 \% \mathrm{CI} 0.64$ to $1.38, \mathrm{P}=0.74$, respectively) or minor allele homozygotes $(\mathrm{HR}=1.10 ; 95 \% \mathrm{CI} 0.50$ to $2.44 ; \mathrm{P}=0.50, \mathrm{HR}=1.22,95 \% \mathrm{CI} 0.51$ to $2.90, \mathrm{P}=0.65$, and $\mathrm{HR}=1.13,95 \% \mathrm{CI}$ 0.28 to $4.53, \mathrm{P}=0.86$, respectively). Similarly, no correlation between genotype and MHEM or ICB was observed when comparing rs12041331 major allele homozygotes to either heterozygotes ( $\mathrm{HR}=0.96$; $95 \%$ CI 0.73 to $1.26 ; \mathrm{P}=0.74$ and $\mathrm{HR}=0.94,95 \%$ CI 0.64 to $1.38, \mathrm{P}=0.74$, respectively) or minor allele 
111 homozygotes $(\mathrm{HR}=0.27 ; 95 \% \mathrm{CI} 0.04$ to $1.93 ; \mathrm{P}=0.19$ and $\mathrm{HR}=1.13,95 \% \mathrm{CI} 0.28$ to $4.53, \mathrm{P}=0.28$, 112 respectively).

113 Using a multivariable Cox regression model, adjusting for age, gender, smoking status, alcohol intake, 114 HDL, LDL-cholesterol, TG, TC, hypertension, BMI, diabetes mellitus and statin use prior to enrolment, 115 we found no evidence of an interaction effect between rs12041331 genotypes and aspirin treatment under 116 the additive or dominant inheritance model, for incident CVD, MACE, ischemic stroke, major 117 haemorrhage and intracranial bleeding (Table 4, Tables S3-8). For calculations of minimal detectable 118 effects, under both models for all endpoints, see Tables S9-10. 


\section{DISCUSSION}

120 There is considerable evidence that the PEARI receptor is a critical part of platelet aggregation in 121 response to several agonists, and that rs12041331 is a strong genetic determinant of platelet 122 aggregation $(5,6,12,23,24)$. However, there is less data regarding the impact of this variant on 123 cardiovascular event risk, in untreated individuals or in patients prescribed antiplatelet medication. In the current study, we sought to evaluate the impact of PEAR1 SNP rs12041331, a well-described genetic variant implicated in aspirin-related platelet function, on MACE and clinically significant bleeding events in a large healthy cohort of elderly individuals randomized to either aspirin or placebo in the ASPREE trial. In analyses of the entire cohort or when stratifying by treatment effect (i.e. aspirin vs. placebo), using either an additive or dominant model of inheritance, we observed no evidence of association for rs12041331 with increased risk of experiencing a thrombotic or bleeding event. Furthermore, we observed no significant interaction effects between PEARl genotype, aspirin use and clinical outcomes using either an additive or dominant model.

Since its identification in 2005, multiple investigations have been published highlighting the role of PEARl in several important biological processes(5). The most extensively studied of these processes has been platelet aggregation; however, other proposed functions of this receptor include maintenance of endothelial cell function(13, 25), megakaryopoiesis and thrombopoiesis(7), neoangiogenesis(26), leukocyte function(17), and neuronal phagocytosis $(27,28)$. These and other publications have prompted numerous genetic studies attempting to correlate PEAR1 polymorphisms with related phenotypes and diseases. From these investigations, rs12041331 has been among the most thoroughly studied, with the strongest effect on platelet-related phenotypes, and established functional effect on PEAR1 expression.

140 Previously, rs12041331 A-allele carriers have been shown to have significantly lower DNA methylation 141 in a CpG-island which contains binding sites for the methylation-sensitive transcription factor CTCF 142 compared to GG homozygotes, presumably leading to differences in gene and protein expression 143 observed by others at this $\operatorname{locus}(11,16)$.

144 Given the effect of rs12041331 on PEAR1 expression and platelet-related processes, recent investigations 145 have focused on the impact of this polymorphism on cardiovascular-related diseases with mixed results. 146 Initial reports in percutaneous coronary intervention patients treated with aspirin and clopidogrel showed that A-allele carriers of this variant experienced MACE $(\mathrm{HR}=2.62 ; 95 \%$ CI $0.96-7.10 ; \mathrm{P}=0.06)$ and cardiovascular-related death $(\mathrm{HR}=3.97 ; 95 \%$ CI $1.10-14.31 ; \mathrm{P}=0.04)$ more frequently compared to $\mathrm{GG}$ homozygotes (29). However, these results were not confirmed in a Flemish population, which reported no association with several different cardiovascular and cerebrovascular phenotypes when using rs12566888 as a proxy SNP for $\operatorname{rs} 12041331\left(\mathrm{R}^{2}=0.99 ; \mathrm{D}^{\prime}=1.00\right.$ with each other $)(30)$. These latter 
findings were consistent with a subsequent publication showing no association between PEARI rs12041331 and ischemic events in Chinese patients with an acute coronary syndrome treated with aspirin and clopidogrel $(\mathrm{HR}=1.30,95 \% \mathrm{CI} 0.74-2.29, \mathrm{P}=0.07)$, although it should be noted that statistical power was likely quite low in this study given a limited sample size of 196 patients(31).

Most recently, $\mathrm{Xu}$ et al. prospectively assessed rs12041331 in over 2,400 Chinese patients with acute coronary syndrome or stable coronary artery disease undergoing stent implantation and treated with aspirin and clopidogrel(32). Based on PEAR1 genotype, they observed significantly different ADPinduced platelet aggregation $(29.1,25.4$, and 22.9 for genotypes GG, GA, and AA, respectively, $\mathrm{P}=0.0005)$ and detected differences in the 30-day incidence of a composite cardiovascular phenotype consisting of cardiovascular death, nonfatal myocardial infarction, and ischemic stroke ( $\mathrm{HR}=2.78,95 \%$ CI $1.13-6.82, \mathrm{P}=0.03)$. Positive associations with deep vein thrombosis in those with sticky platelet syndrome as well as coronary artery aneurysm in individuals with Kawasaki disease have also been shown by PEAR1 rs12041331 genotype(33, 34).

165 Further studies have suggested a genotype $\mathrm{x}$ aspirin interaction exists whereby aspirin-treated carriers of 166 rs12041331 minor (A) allele have enhanced risk of experiencing a clinical event compared to carriers of 167 the same allele who are not treated with aspirin(29). Specifically, it was observed in stable coronary 168 artery disease patients of European descent that rs12041331 A-allele carriers treated with aspirin have 169 significantly increased risk of myocardial infarction (odds ratio $[\mathrm{OR}]=2.08$, 95\% CI $1.01-4.09$; $170 \mathrm{P}=0.048)$ compared to those who were not exposed to aspirin $(\mathrm{OR}=0.25,95 \%$ CI $0.03-2.03, \mathrm{P}=0.19)$. 171 Similar trends were observed using a composite cardiovascular outcome consisting of death, non-fatal 172 stroke, and non-fatal myocardial infarction $(\mathrm{OR}=1.62,95 \% \mathrm{CI} 0.91-2.90, \mathrm{P}=0.10$ and $\mathrm{OR}=0.54,95 \%$ 173 CI $0.22-1.31, \mathrm{P}=0.16$, respectively)(29). Evidence also suggests that the PEAR1 receptor may be able to 174 exert cardiovascular-related effects through processes that are independent of platelet function (e.g. 175 endothelial-related effects). Investigation of other molecular processes that are regulated by PEAR1 would likely allow for more targeted investigation of effects that may modify cardiovascular risk.

177 It is noteworthy that all previous studies discussed above have been undertaken in populations known to 178 be affected by cardiovascular disease. The present study, however, has been undertaken in a highly 179 selected population of healthy older individuals with no previous diagnosis of atherothrombotic 180 cardiovascular disease, and in the context of primary prevention. Our study results, therefore, must be 181 interpreted accordingly.

182 In our investigation, which is the largest study of PEARI rs12041331 and cardiovascular outcomes 183 conducted to date, we did not observe significant association between rs12041331 and cardiovascular 
events or clinically significant bleeding in the entire cohort, regardless of aspirin use (Table 3). We also found no significant effects in interaction analyses using either the additive (Table 4, Tables S3-7) or dominant (Table S8) genetic model. Given that the minor allele of this variant resulted in lower agoniststimulated platelet aggregation in prior investigations, we also assessed whether aspirin-dependent effects

188 could modify the relationship between PEARI genotype and clinically significant bleeding events. 189 Consistent with our analyses pertaining to thrombotic outcomes, no association was observed between 190 rs12041331 and bleeding in both placebo- or aspirin-randomized individuals and no statistically 191 significant interaction was observed between aspirin treatment and genotype on occurrence of bleeding 192 events.

193 While our data suggests that this polymorphism is not an important contributor to clinical events in a 194 healthy older population, in the setting of primary prevention, additional studies are warranted, investigating the effect of rs12041331 on MACE in other populations, and including the general population, and patients with higher cardiovascular burden, such as those following a coronary 197 intervention procedure, and/or on a dual antiplatelet therapy regimen.

198 Strengths of our study include the sample size, being the largest evaluation of PEARI rs12041331 conducted to date, with regards to cardiovascular outcomes and aspirin use. To our knowledge, it is also the only study to examine the effect of rs12041331 on clinically significant bleeding events. In addition, the prospective, randomized nature of the study design implemented in the ASPREE trial allowed us to confidently assess the interaction between PEARl genotype and aspirin use on adverse events in cohorts that were well-matched for clinical and anthropometric characteristics known to influence cardiovascular 205 evaluation of suspected outcomes and ultimately allowed us to maximize data consistency while minimizing potential biases.

207 Limitations of our study include the highly selected nature of the ASPREE population, and the 208 predominance of European ancestry, which limit the generalizability of our results to other populations. 209 Participants enrolled into the ASPREE trial were elderly, generally healthy individuals with no known 210 atherothrombotic cardiovascular disease and were randomized to aspirin in the context of primary 211 prevention. Therefore, our study results may not be indicative of data obtained in patients with more 212 severe cardiovascular burden and/or in the context of secondary prevention. Furthermore, ethnicity213 specific analyses were not performed in the current investigation. While prior investigations in European214 , African- and Asian-derived populations have shown consistent effects of PEAR1 rs12041331 genotype 215 with regards to platelet aggregation, the vast majority of ASPREE participants were Caucasian ( $93 \%)$. 216 Therefore, caution should be used when extrapolating these results to populations of other ethnic/racial 
origins especially given that the minor allele frequency of rs12041331 varies significantly among such groups. Our study had very few events in the AA homozygote group, which limited the interpretation of results from the additive genetic model used in the primary analysis. To account for this, we conducted analysis using a dominant model, and found similar results. Finally, it is important to note that participants were randomized to low dose aspirin in this study (i.e. $100 \mathrm{mg} /$ daily). If a true interaction between PEARl genotype and aspirin use exists, it is possible that such an interaction may be dependent on aspirin dose.

224 It is also noteworthy that low-dose aspirin did not significantly decrease CVD, MACE, or stroke events 225 versus placebo in the overall ASPREE trial (21). The lack of an aspirin treatment effect in the ASPREE 226 healthy elderly population must be considered when interpreting of the lack of associations found in 227 genetic analysis of PEAR1 rs12041331 in this cohort. It is possible that the selection bias that occurred as 228 a result of the strict ASPREE enrolment criteria, excluding any individuals with a history of diagnosed 229 cardiovascular events, has resulted in ascertainment of a unique population where genetic effects are 230 diminished or attenuated. This is indeed consistent with others studies that have shown a diminished 231 effect of genetic risk factors as a function of age in other contexts $(35,36)$.

232 Based on our calculations of minimal detectable differences between rs12041331 genotypes and the 233 endpoints analysed in the ASPREE trial (Tables S9-10), it is possible that larger study populations or 234 meta-analyses may uncover significant effects of PEARI and aspirin use on cardiovascular or bleeding 235 events. However, it is also possible that in such larger study populations, investigations may find the 236 effect of rs12041331 moving closer towards zero. Further post-hoc analysis of other large aspirin trials is 237 therefore warranted, as new datasets from other large trials become available.

238 In conclusion, we could not replicate previous findings suggesting that PEAR1 rs12041331 was an 239 important genetic determinant of cardiovascular outcomes. Furthermore, no effects of this variant on 240 clinically significant bleeding was observed in the ASPREE study. While differences in study designs 241 and participant populations may, in part, explain these divergent results, our data do provide robust 242 evidence that genetic variation in PEARI do not contribute to cardiovascular disease risk in relatively 243 healthy older individuals regardless of aspirin treatment. Additional well-powered investigations in 244 patients with high-risk clinical indications would likely be helpful to determine the role, if any, PEARI 245 has on the occurrence of adverse clinical events.

This article is protected by copyright. All rights reserved 


\section{STUDY HIGHLIGHTS:}

247 o What is the current knowledge on the topic?

248 Identification of genetic factors that influence aspirin response may facilitate more personalized

249 antiplatelet treatment, and targeted prevention of cardiovascular disease. Yet identification of

250 pharmacogenomic variants of high clinical effect for aspirin has been challenging. Previous studies

251 suggest that genetic variation in the platelet endothelial aggregation receptor 1 (PEAR1) gene results in

252 altered aspirin efficacy. However, association of PEAR1 genotypes with cardiovascular events is less

253 clear.

254

255 o What question did this study address?

256 Our study, the largest study of PEARI and cardiovascular outcomes to date, examined the effect of

257 rs 12041331 genotypes and aspirin use on cardiovascular and bleeding events in a population of 13,547

258 healthy older individuals without a history of atherothrombotic cardiovascular disease enrolled in the

259 ASPirin in Reducing Events in the Elderly (ASPREE) trial.

260

261 o What does this study add to our knowledge?

262 We observed no association between genotypes and these outcomes, and no interaction with aspirin use in 263 the ASPREE population. Our study indicates PEAR1 genetic variation does not influence aspirin primary 264 prevention in healthy older individuals without a history of cardiovascular events.

265

266 o How might this change clinical pharmacology or translational science?

267 Our study represents an important finding that contributes to the evolving pharmacogenomics evidence268 base for aspirin, one of the world's most widely used drugs.

This article is protected by copyright. All rights reserved 


\section{ACKNOWLEDGEMENTS}

271 We thank the trial staff in Australia and the United States, the participants who volunteered for this trial, 272 and the general practitioners and staff of the medical clinics who cared for the participants.

\section{AUTHOR CONTRIBUTIONS}

274 P.L and J.P.L wrote the manuscript. J.P.L, A.R.S, J.J.M and P.L made substantial contributions to the 275 conception and design of the work. M.R, S.X, G.P, R.W, M.N, A.M.T, C.M.R and A.M.M made 276 substantial contributions to the acquisition, analysis, or interpretation of data, and drafting and revising of 277 the manuscript. J.J.M, A.M.M and P.L are accountable for all aspects of the work in ensuring that 278 questions related to the accuracy or integrity of any part of the work are appropriately investigated and 279 resolved.

This article is protected by copyright. All rights reserved 


\section{REFERENCES}

(1) Becker, D.M. et al. Sex differences in platelet reactivity and response to low-dose aspirin therapy. JAMA 295, 1420-7 (2006).

(2) Hankey, G.J. \& Eikelboom, J.W. Aspirin resistance. Lancet 367, 606-17 (2006).

(3) Larsen, S.B., Grove, E.L., Neergaard-Petersen, S., Wurtz, M., Hvas, A.M. \& Kristensen, S.D. Determinants of reduced antiplatelet effect of aspirin in patients with stable coronary artery disease. PLoS One 10, e0126767 (2015).

(4) Faraday, N. et al. Heritability of platelet responsiveness to aspirin in activation pathways directly and indirectly related to cyclooxygenase-1. Circulation 115, 2490-6 (2007).

(5) Nanda, N. et al. Platelet endothelial aggregation receptor 1 (PEAR1), a novel epidermal growth factor repeat-containing transmembrane receptor, participates in platelet contact-induced activation. J Biol Chem 280, 24680-9 (2005).

(6) Kauskot, A., Di Michele, M., Loyen, S., Freson, K., Verhamme, P. \& Hoylaerts, M.F. A novel mechanism of sustained platelet alphallbbeta3 activation via PEAR1. Blood 119, 4056-65 (2012).

(7) Kauskot, A. et al. PEAR1 attenuates megakaryopoiesis via control of the PI3K/PTEN pathway. Blood 121, 5208-17 (2013).

(8) Johnson, A.D. et al. Genome-wide meta-analyses identifies seven loci associated with platelet aggregation in response to agonists. Nat Genet 42, 608-13 (2010).

(9) Qayyum, R. et al. Genome-wide association study of platelet aggregation in African Americans. BMC Genet 16, 58 (2015).

(10) Herrera-Galeano, J.E. et al. A novel variant in the platelet endothelial aggregation receptor-1 gene is associated with increased platelet aggregability. Arterioscler Thromb Vasc Biol 28, 148490 (2008).

(11) Faraday, N. et al. Identification of a specific intronic PEAR1 gene variant associated with greater platelet aggregability and protein expression. Blood 118, 3367-75 (2011).

(12) Keramati, A.R. et al. Targeted deep sequencing of the PEAR1 locus for platelet aggregation in European and African American families. Platelets 30, 380-6 (2019).

(13) Lewis, J.P. et al. Pharmacogenomic Polygenic Response Score Predicts Ischemic Events and Cardiovascular Mortality in Clopidogrel-Treated Patients. Eur Heart J Cardiovasc Pharmacother, (2019).

(14) Li, M. et al. Association of PEAR1 rs12041331 polymorphism and pharmacodynamics of ticagrelor in healthy Chinese volunteers. Xenobiotica 47, 1130-8 (2017). 
(15) Xiang, Q., Cui, Y., Zhao, X. \& Zhao, N. Identification of PEAR1 SNPs and their influences on the variation in prasugrel pharmacodynamics. Pharmacogenomics 14, 1179-89 (2013).

(16) Izzi, B. et al. Allele-specific DNA methylation reinforces PEAR1 enhancer activity. Blood 128, 1003-12 (2016).

(17) Izzi, B. et al. Variation of PEAR1 DNA methylation influences platelet and leukocyte function. Clin Epigenetics 11, 151 (2019).

(18) McNeil, J.J. et al. Baseline Characteristics of Participants in the ASPREE (ASPirin in Reducing Events in the Elderly) Study. J Gerontol A Biol Sci Med Sci 72, 1586-93 (2017).

(19) McNeil, J.J. et al. Effect of Aspirin on All-Cause Mortality in the Healthy Elderly. N Engl J Med 379, 1519-28 (2018).

(20) McNeil, J.J. et al. Effect of Aspirin on Disability-free Survival in the Healthy Elderly. N Engl J Med 379, 1499-508 (2018).

(21) McNeil, J.J. et al. Effect of Aspirin on Cardiovascular Events and Bleeding in the Healthy Elderly. N Engl J Med 379, 1509-18 (2018).

(22) Das, S. et al. Next-generation genotype imputation service and methods. Nat Genet 48, 1284-7 (2016).

(23) Vandenbriele, C. et al. Dextran sulfate triggers platelet aggregation via direct activation of PEAR1. Platelets 27, 365-72 (2016).

(24) Xiang, Q., Zhou, S., Lewis, J.P., Shuldiner, A.R., Ren, G. \& Cui, Y. Genetic Variants of PEAR1 are Associated with Platelet Function and Antiplatelet Drug Efficacy: A Systematic Review and MetaAnalysis. Curr Pharm Des 23, 6815-27 (2017).

(25) Zhan, Q., Ma, X. \& He, Z. PEAR1 suppresses the proliferation of pulmonary microvascular endothelial cells via PI3K/AKT pathway in ALI model. Microvasc Res 128, 103941 (2020).

(26) Vandenbriele, C. et al. Platelet endothelial aggregation receptor-1: a novel modifier of neoangiogenesis. Cardiovasc Res 108, 124-38 (2015).

(27) Wu, H.H. et al. Glial precursors clear sensory neuron corpses during development via Jedi-1, an engulfment receptor. Nat Neurosci 12, 1534-41 (2009).

(28) Sullivan, C.S. et al. The adaptor protein GULP promotes Jedi-1-mediated phagocytosis through a clathrin-dependent mechanism. Mol Biol Cell 25, 1925-36 (2014).

(29) Lewis, J.P. et al. Genetic variation in PEAR1 is associated with platelet aggregation and cardiovascular outcomes. Circ Cardiovasc Genet 6, 184-92 (2013). 
(30) Yang, W.Y. et al. PEAR1 is not a major susceptibility gene for cardiovascular disease in a Flemish population. BMC Med Genet 18, 45 (2017).

(31) Nie, X.Y. et al. Genetic mutations in PEAR1 associated with cardiovascular outcomes in Chinese patients with acute coronary syndrome. Thromb Res 163, 77-82 (2018).

(32) Xu, K. et al. Impact of Platelet Endothelial Aggregation Receptor-1 Genotypes on Platelet Reactivity and Early Cardiovascular Outcomes in Patients Undergoing Percutaneous Coronary Intervention and Treated With Aspirin and Clopidogrel. Circ Cardiovasc Interv 12, e007019 (2019).

(33) Sokol, J., Skerenova, M., Ivankova, J., Simurda, T. \& Stasko, J. Association of Genetic Variability in Selected Genes in Patients With Deep Vein Thrombosis and Platelet Hyperaggregability. Clin Appl Thromb Hemost 24, 1027-32 (2018).

(34) Pi, L. et al. A PEAR1 polymorphism (rs12041331) is associated with risk of coronary artery aneurysm in Kawasaki disease. Ann Hum Genet 83, 54-62 (2019).

(35) Kuchenbaecker, K.B. et al. Risks of Breast, Ovarian, and Contralateral Breast Cancer for BRCA1 and BRCA2 Mutation Carriers. JAMA 317, 2402-16 (2017).

(36) Moller, P. et al. Cancer risk and survival in path_MMR carriers by gene and gender up to 75 years of age: a report from the Prospective Lynch Syndrome Database. Gut 67, 1306-16 (2018).

This article is protected by copyright. All rights reserved 


\section{FIGURE LEGENDS}

Figure 1. Schematic Overview (Graphical Abstract). We examined the relationship between rs12041331 genotypes and cardiovascular and bleeding events in a randomised placebo-controlled study population of $\mathrm{N}=13,547$ participants of the ASPirin in Reducing Events in the Elderly (ASPREE) trial. Participants had no previous diagnosis of atherothrombotic cardiovascular disease at enrolment, and were randomized to either $100 \mathrm{mg}$ daily low-dose aspirin or placebo for a median 4.7 years of follow-up. Endpoints analysed included the primary cardiovascular disease endpoint in the ASPREE study (CVD), and composites of major adverse cardiovascular events (MACE) and ischaemic stroke; and bleeding events including major hemorrhage and intracranial bleeding. Details of ASPREE endpoints are described previously (21). The modifying effect of the rs12041331-A genotype on association with cardiovascular and bleeding events and aspirin treatment was analysed using an interaction term in a multivariable Cox regression model.

This article is protected by copyright. All rights reserved 


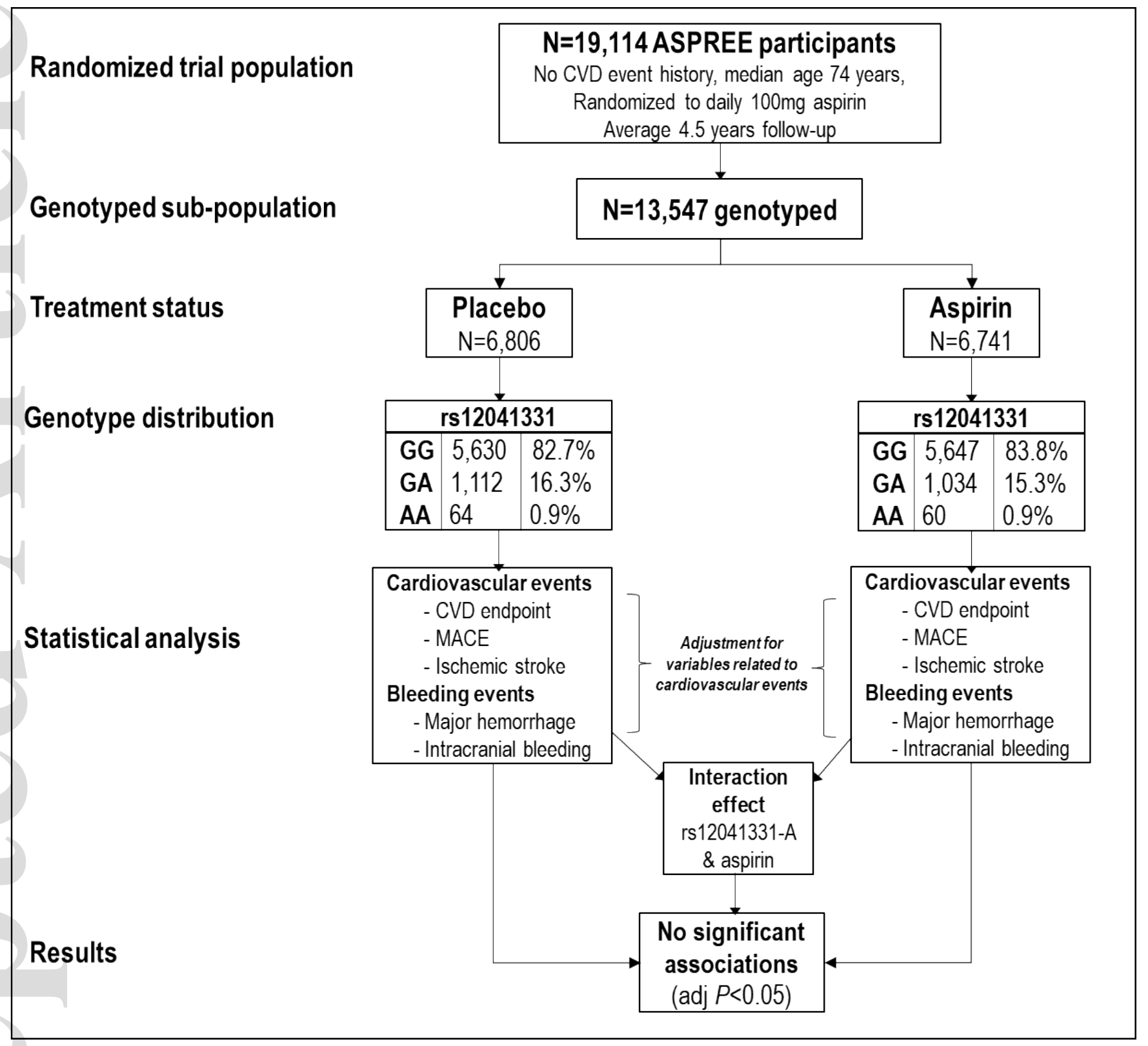

This article is protected by copyright. All rights reserved 
Figure 2: Cumulative incidence curves (additive genetic model). We undertook cumulative incidence analysis of cardiovascular and bleeding events in aspirin and placebo groups, stratified by rs12041331 genotypes using an additive model of genetic inheritance; GG wild type (blue curves), AG heterozygotes (red curves), and AA homozygotes (yellow curves). Events analysed included the primary cardiovascular disease endpoint in the ASPREE study (CVD) (a-b), and composites of major adverse cardiovascular events (MACE) (c-d) and ischaemic stroke (STROKE) (e-f); and the ASPREE clinically significant bleeding endpoint sub-comp (i-j).
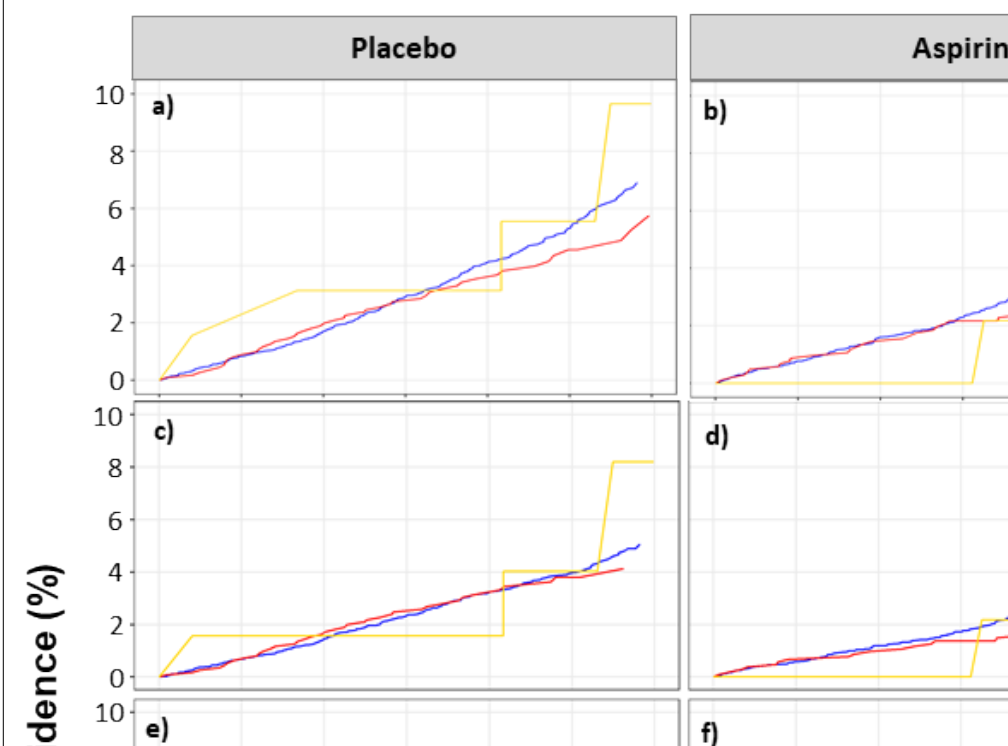

(36)

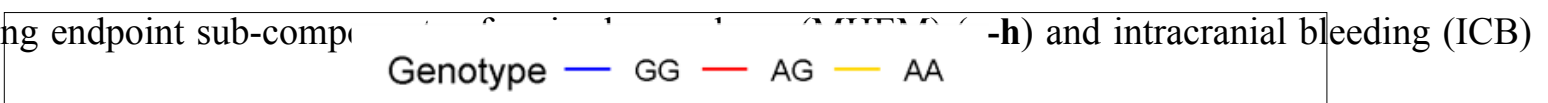


Figure 3: Cumulative incidence curves (dominant genetic model). We undertook cumulative

371 incidence analysis of cardiovascular and bleeding events in aspirin and placebo groups, stratified by

372 rs 12041331 genotypes using a dominant model of genetic inheritance; GG wild type (blue curves) versus

373 AG/AA carriers (red curves). Events analysed included the primary cardiovascular disease endpoint in the

374 ASPREE study (CVD) (a-b), and composites of major adverse cardiovascular events (MACE) (c-d) and

375 ischaemic stroke (STROKE) (e-f); and the ASPREE clinically significant bleeding endpoint sub-

376 components of major hemorrhage (MHEM) (g-h) and intracranial bleeding (ICB) (i-j).

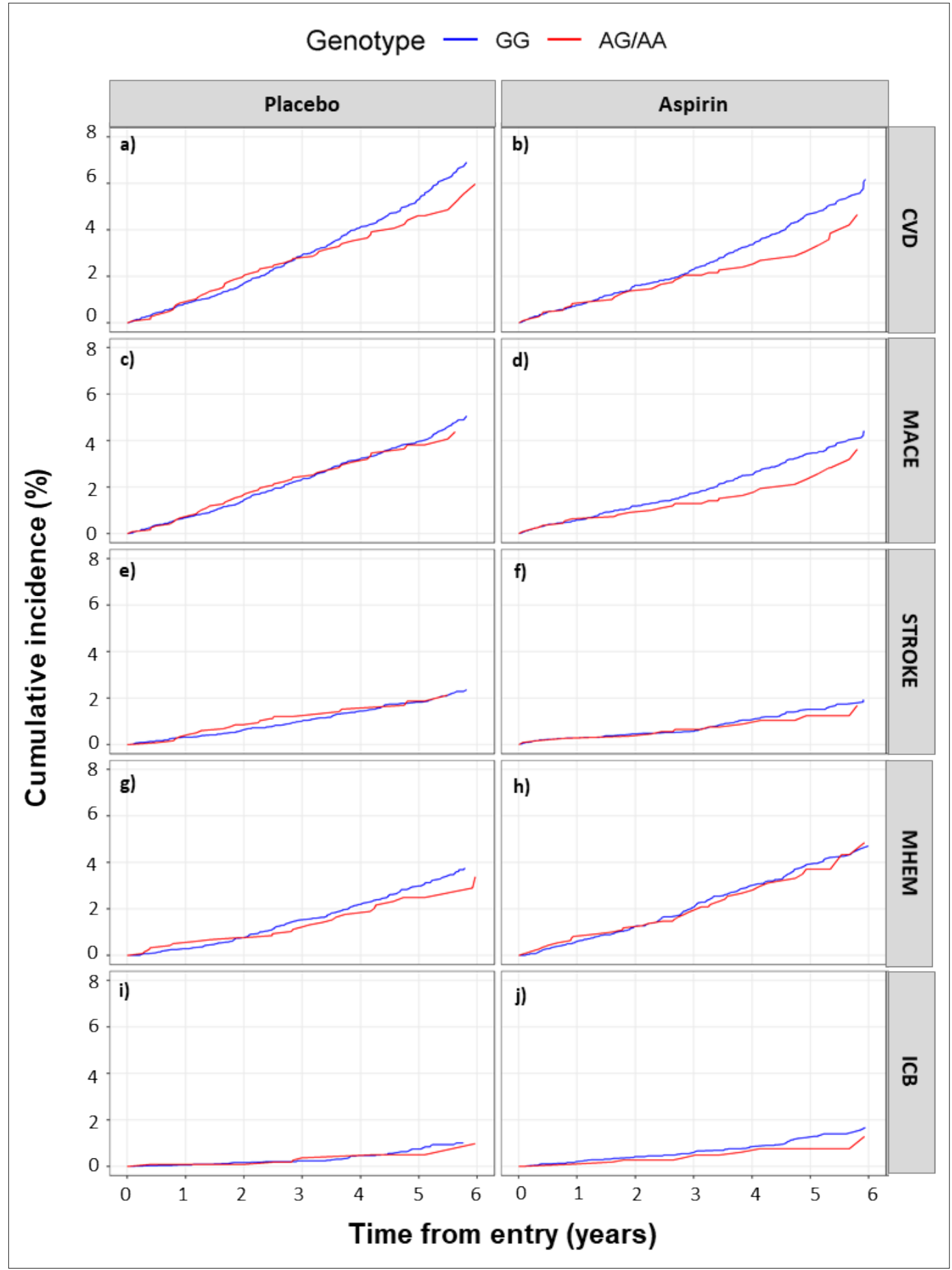


Table 1: Characteristics of Genotyped Participants by Treatment Status. Data summaries are median (IQR) for continuous measures, and n (\%) for categorical measures. *Self-report

\begin{tabular}{|c|c|c|c|}
\hline & $\begin{array}{l}\text { Placebo } \\
\mathrm{N}=\mathbf{6 , 8 0 6}\end{array}$ & $\begin{array}{l}\text { Aspirin } \\
\mathrm{N}=6,741\end{array}$ & $\begin{array}{c}\text { Total } \\
\mathrm{N}=\mathbf{1 3 , 5 4 7}\end{array}$ \\
\hline Age at randomization (yrs) & $73.9(71.7,77.3)$ & $73.9(71.7,77.3)$ & $73.9(71.7,77.3)$ \\
\hline $65-73.9 \mathrm{yr}$ & $3,485(51.2 \%)$ & $3,446(51.1 \%)$ & $6,931(51.2 \%)$ \\
\hline$\geq 74 \mathrm{yr}$ & $3,321(48.8 \%)$ & $3,295(48.9 \%)$ & $6,616(48.8 \%)$ \\
\hline \multicolumn{4}{|l|}{ Gender } \\
\hline Female & $3,716(54.6 \%)$ & $3,691(54.8 \%)$ & $7,407(54.7 \%)$ \\
\hline Male & $3,090(45.4 \%)$ & $3,050(45.2 \%)$ & $6,140(45.3 \%)$ \\
\hline \multicolumn{4}{|l|}{$\begin{array}{l}\text { Blood lipid levels } \\
\text { (mg/DL at baseline) }\end{array}$} \\
\hline LDL-C & $119.9(96.7,140.0)$ & $116.0(96.0,139.2)$ & $116.0(96.7$ to 139.2$)$ \\
\hline HDL-C & $58.0(50.3,69.6)$ & $58.0(50.0,69.6)$ & $58.0(50.3$ to 69.6$)$ \\
\hline $\mathrm{TG}$ & $106.3(79.7,141.7)$ & $106.3(79.7,141.7)$ & $106.3(79.7$ to 141.7$)$ \\
\hline $\mathrm{TC}$ & $201.1(177.9,228.2)$ & $201.1(177.9,228.2)$ & 201.1 (177.9 to 228.2 ) \\
\hline BMI kg/m² (baseline) & $27.4(24.9-30.5)$ & $27.4(24.9-30.5)$ & $27.4(24.9$ to 30.5$)$ \\
\hline Underweight, $<20$ & $112(1.7 \%)$ & $119(1.8 \%)$ & $231(1.7 \%)$ \\
\hline Normal, 20-24.9 & $1,635(24.1 \%)$ & $1,643(24.5 \%)$ & $3,278(24.3 \%)$ \\
\hline Overweight, 25-29.9 & $3,112(45.9 \%)$ & $3,037(45.2 \%)$ & $6,149(45.6 \%)$ \\
\hline Obese, $\geq 30$ & $1,917(28.3 \%)$ & $1,914(28.5 \%)$ & $3,831(28.4 \%)$ \\
\hline Statin use* & $2,369(34.8 \%)$ & $2,384(35.4 \%)$ & $4,753(35.1 \%)$ \\
\hline Hypertension* & $5,056(74.3 \%)$ & $4,928(73.1 \%)$ & $9,984(73.7 \%)$ \\
\hline
\end{tabular}

This article is protected by copyright. All rights reserved 
Table 2. Genotype Distribution by Treatment Status. Genome-wide SNP genotyping of N=14,177 samples collected by the ASPREE Healthy Ageing Biobank was performed using the Axiom 2.0 Precision Medicine Diversity Research Array (Thermo Fisher Scientific, CA, USA) following standard protocols. A total of $\mathrm{N}=13547$ samples passed post-genotyping quality control (QC) filters. Samples were 382 excluded based on filters related to gender discordance, genotyping rate $(<95 \%)$, relatedness, Hardy383 Weinberg equilibrium, and ethnicity. Imputation was performed on European-ancestry samples using the 384 haplotype reference consortium (HRC) panel on the Michigan imputation server (22). Post-imputation 385 QC removed all variants with $<0.3$ imputation quality scores. The imputation quality score of the variant 386 rs12041331 was $\mathrm{R}^{2}=0.99$.

\begin{tabular}{|c|c|c|c|}
\hline rs12041331 & PLACEBO & ASPIRIN & TOTAL \\
\hline \multirow{2}{*}{ GG } & 5,630 & 5,647 & 11,277 \\
& $(82.7 \%)$ & $(83.8 \%)$ & $(83.2 \%)$ \\
\hline \multirow{2}{*}{ GA } & 1,112 & 1,034 & 2,146 \\
& $(16.3 \%)$ & $(15.3 \%)$ & $(15.8 \%)$ \\
\hline \multirow{2}{*}{ AA } & 64 & 60 & 124 \\
& $(0.9 \%)$ & $(0.9 \%)$ & $\mathbf{1 3 , 5 4 7}$ \\
\hline
\end{tabular}

This article is protected by copyright. All rights reserved 
Table 3: Association of rs12041331 Genotypes with ASPREE Cardiovascular Disease and Bleeding Events in Aspirin and Placebo Treatment Groups. We used Cox proportional hazard regression to model the relationship between rs12041331 genotypes with cardiovascular and bleeding events. Endpoints analysed included the ASPREE cardiovascular disease endpoint (CVD), and sub-components of major adverse cardiovascular events (MACE) and ischaemic stroke (STROKE); and the ASPREE clinically significant bleeding endpoint (CSB), and sub-components of major hemorrhage (MHEM) and intracranial bleeding (ICB). Details of ASPREE endpoints are described previously (21). HR adj: Hazard ratio, adjusted for age, gender, smoking status, alcohol intake, high density lipoproteins, low density lipoprotein cholesterol, triglycerides, total cholesterol, hypertension, body mass index, diabetes mellitus and statin use prior to enrolment (multivariable Cox regression analysis).

\begin{tabular}{|c|c|c|c|c|c|c|c|c|c|}
\hline \multicolumn{10}{|c|}{ ASPREE Cardiovascular Disease Endpoint (CVD) } \\
\hline \multicolumn{5}{|c|}{ PLACEBO } & \multicolumn{5}{|c|}{ ASPIRIN } \\
\hline 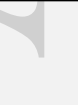 & $\begin{array}{c}\text { NO CVD } \\
\text { n (\%) }\end{array}$ & $\begin{array}{l}\text { CVD } \\
\text { n (\%) }\end{array}$ & $\begin{array}{c}\text { HR adj } \\
(95 \% \text { CI })\end{array}$ & $p$-value & & $\begin{array}{c}\text { NO CVD } \\
\text { n (\%) }\end{array}$ & $\begin{array}{l}\text { CVD } \\
\text { n (\%) }\end{array}$ & $\begin{array}{c}\text { HR adj } \\
(95 \% \text { CI })\end{array}$ & $p$-value \\
\hline GG & $\begin{array}{c}5351 \\
(95.0)\end{array}$ & $\begin{array}{l}279 \\
(5.0)\end{array}$ & 1 & - & GG & $5406(95.7)$ & $\begin{array}{l}241 \\
(4.3)\end{array}$ & 1 & - \\
\hline GA & $\begin{array}{c}1065 \\
(95.8)\end{array}$ & $\begin{array}{c}47 \\
(4.2)\end{array}$ & $\begin{array}{c}0.84 \\
(0.61,1.15)\end{array}$ & 0.27 & GA & $1001(96.8)$ & $\begin{array}{c}33 \\
(3.2)\end{array}$ & $\begin{array}{c}0.76 \\
(0.53,1.09)\end{array}$ & 0.14 \\
\hline $\mathbf{A A}$ & $\begin{array}{c}60 \\
(93.8)\end{array}$ & $\begin{array}{c}4 \\
(6.3)\end{array}$ & $\begin{array}{c}1.28 \\
(0.48,3.40)\end{array}$ & 0.62 & $\mathbf{A A}$ & $58(96.7)$ & $\begin{array}{c}2 \\
(3.3)\end{array}$ & $\begin{array}{c}0.82 \\
(0.21,3.21)\end{array}$ & 0.78 \\
\hline \multicolumn{10}{|c|}{ Major Adverse Cardiovascular Events (MACE) } \\
\hline \multicolumn{5}{|c|}{ PLACEBO } & \multicolumn{5}{|c|}{ ASPIRIN } \\
\hline & $\begin{array}{c}\text { NO } \\
\text { MACE } \\
\text { n (\%) }\end{array}$ & $\begin{array}{l}\text { MACE } \\
\text { n (\%) }\end{array}$ & $\begin{array}{c}\text { HR adj } \\
(95 \% \text { CI) }\end{array}$ & $p$-value & & $\begin{array}{c}\text { NO MACE } \\
\text { n (\%) }\end{array}$ & $\begin{array}{l}\text { MACE } \\
\text { n (\%) }\end{array}$ & $\begin{array}{c}\text { HR adj } \\
(95 \% \mathrm{CI})\end{array}$ & $p$-value \\
\hline GG & $\begin{array}{c}5418 \\
(96.2)\end{array}$ & $212(3.8)$ & 1 & - & GG & $5469(96.8)$ & $178(3.2)$ & 1 & - \\
\hline GA & $\begin{array}{c}1073 \\
(96.5)\end{array}$ & $39(3.5)$ & $\begin{array}{c}0.93 \\
(0.66,1.31)\end{array}$ & 0.66 & GA & $1011(97.8)$ & $23(2.2)$ & $\begin{array}{c}0.70 \\
(0.46,1.09)\end{array}$ & 0.11 \\
\hline $\mathbf{A A}$ & $61(95.3)$ & $4(6.3)$ & $\begin{array}{c}1.22 \\
(0.40,3.71)\end{array}$ & 0.73 & $\mathbf{A A}$ & $58(96.7)$ & $2(3.3)$ & $\begin{array}{c}1.15 \\
(0.29,4.54)\end{array}$ & 0.85 \\
\hline \multicolumn{10}{|c|}{ Ischemic Stroke (STROKE) } \\
\hline \multicolumn{5}{|c|}{ PLACEBO } & \multicolumn{5}{|c|}{ ASPIRIN } \\
\hline
\end{tabular}

This article is protected by copyright. All rights reserved 


\begin{tabular}{|c|c|c|c|c|c|c|c|c|c|}
\hline & $\begin{array}{c}\text { NO } \\
\text { STROKE } \\
\text { n (\%) }\end{array}$ & $\begin{array}{c}\text { STROKE } \\
\text { n (\%) }\end{array}$ & $\begin{array}{c}\text { HR adj } \\
(95 \% \text { CI) }\end{array}$ & $p$-value & & $\begin{array}{c}\text { NO } \\
\text { STROKE } \\
\text { n (\%) }\end{array}$ & $\begin{array}{c}\text { STROKE } \\
\text { n (\%) }\end{array}$ & $\begin{array}{c}\text { HR adj } \\
(95 \% \text { CI) }\end{array}$ & $p$-value \\
\hline GG & $\begin{array}{l}5534 \\
(98.3)\end{array}$ & $\begin{array}{c}96 \\
(1.7)\end{array}$ & 1 & - & GG & $\begin{array}{c}5773 \\
(98.7)\end{array}$ & $\begin{array}{c}74 \\
(1.3)\end{array}$ & 1 & - \\
\hline $\mathbf{A A}$ & $\begin{array}{c}63 \\
(98.4)\end{array}$ & $\begin{array}{c}1 \\
(1.6)\end{array}$ & $\begin{array}{c}0.94 \\
(0.13,6.63)\end{array}$ & 0.95 & $\mathbf{A A}$ & $\begin{array}{c}59 \\
(98.3)\end{array}$ & $\begin{array}{c}1 \\
(1.7)\end{array}$ & $\begin{array}{c}1.36 \\
(0.19,9.83)\end{array}$ & 0.76 \\
\hline \multicolumn{10}{|c|}{ Major Hemorrhage (MHEM) } \\
\hline & $\begin{array}{c}\text { NO } \\
\text { MHEM } \\
\text { n (\%) }\end{array}$ & $\begin{array}{c}\text { МНЕМ } \\
\text { n (\%) }\end{array}$ & $\begin{array}{c}\text { HR adj } \\
(95 \% \text { CI) }\end{array}$ & $p$-value & & $\begin{array}{c}\text { NO MHEM } \\
\text { n }(\%)\end{array}$ & $\begin{array}{c}\text { MHEM } \\
\text { n (\%) }\end{array}$ & $\begin{array}{c}\text { HR adj } \\
(95 \% \text { CI) }\end{array}$ & $p$-value \\
\hline GG & $\begin{array}{l}5479 \\
(97.3)\end{array}$ & $96(1.7)$ & 1 & & GG & $5448(96.5)$ & $199(3.5)$ & 1 & - \\
\hline GA & $\begin{array}{l}1093 \\
(98.3)\end{array}$ & $19(1.7)$ & $\begin{array}{c}0.88 \\
(0.57,1.34)\end{array}$ & 0.57 & GA & $\begin{array}{c}998 \\
(96.5)\end{array}$ & $\begin{array}{c}36 \\
(3.5)\end{array}$ & $\begin{array}{c}1.02 \\
(0.71,1.45)\end{array}$ & 0.92 \\
\hline $\mathbf{A A}$ & $\begin{array}{c}63 \\
(98.4)\end{array}$ & $\begin{array}{c}1 \\
(1.6)\end{array}$ & $\begin{array}{c}0.60 \\
(0.08,4.25)\end{array}$ & 0.61 & $\mathbf{A A}$ & $\begin{array}{c}60 \\
(100.0)\end{array}$ & $\begin{array}{c}0 \\
(0)\end{array}$ & NA & - \\
\hline & $\begin{array}{c}\text { NO ICB } \\
\text { n }(\%)\end{array}$ & $\begin{array}{c}\text { ICB } \\
\text { n (\%) }\end{array}$ & $\begin{array}{c}\text { HR adj } \\
(95 \% \mathrm{CI})\end{array}$ & $p$-value & & $\begin{array}{r}\text { NO ICB } \\
\text { n }(\%)\end{array}$ & $\begin{array}{l}\text { ICB } \\
\text { n (\%) }\end{array}$ & $\begin{array}{c}\text { HR adj } \\
(95 \% \text { CI) }\end{array}$ & $p$-value \\
\hline GG & $\begin{array}{l}5594 \\
(99.4)\end{array}$ & $36(0.6)$ & 1 & - & GG & $5585(98.9)$ & $62(1.1)$ & 1 & - \\
\hline GA & $\begin{array}{l}1106 \\
(99.5)\end{array}$ & $6(0.5)$ & $\begin{array}{c}1.03 \\
(0.63,1.68)\end{array}$ & 0.92 & GA & $1026(99.2)$ & $8(0.8)$ & $\begin{array}{c}0.81 \\
(0.43,1.52)\end{array}$ & 0.51 \\
\hline $\mathbf{A A}$ & $63(98.4)$ & $1(1.6)$ & $\begin{array}{c}0.94 \\
(0.13,6.63)\end{array}$ & 0.95 & $\mathbf{A A}$ & $60(100.0)$ & $0(0)$ & - & - \\
\hline
\end{tabular}

This article is protected by copyright. All rights reserved 
Table 4. Tests of Interaction Effect between treatment group and the rs12041331-A Genotype on ASPREE Cardiovascular Disease and Bleeding Events. The modifying effect of the rs12041331-A genotype on association between incident CVD events and aspirin treatment was analysed using an interaction term in a multivariable Cox regression model using participants from both groups, adjusted for age, gender, smoking status, alcohol intake, high density lipoproteins, low density lipoprotein cholesterol, triglycerides, total cholesterol, hypertension, body mass index, diabetes mellitus and statin use prior to enrolment. Endpoints analysed included the ASPREE cardiovascular disease endpoint (CVD), and sub-components of major adverse cardiovascular events (MACE) and ischaemic stroke (STROKE); and the ASPREE clinically significant bleeding endpoint (CSB), and sub-components of major hemorrhage (MHEM) and intracranial bleeding (ICB). Details of ASPREE endpoints are described previously (21).

\begin{tabular}{|c|c|c|c|c|c|c|c|c|c|c|}
\hline rs12041331 & \multicolumn{2}{|c|}{ CVD } & \multicolumn{2}{c|}{ MACE } & \multicolumn{2}{c|}{ STROKE } & \multicolumn{2}{c|}{ MHEM } & \multicolumn{2}{c|}{ ICB } \\
\hline & $\mathbf{z}$ & $P$ & $\mathbf{z}$ & $\boldsymbol{P}$ & $\mathbf{z}$ & $\boldsymbol{P}$ & $\mathbf{z}$ & $\boldsymbol{P}$ & $\mathrm{z}$ & $P$ \\
\hline $\begin{array}{c}\text { GA \# } \\
\text { Aspirin }\end{array}$ & -0.46 & 0.649 & -0.99 & 0.321 & -0.58 & 0.561 & 0.54 & 0.591 & -0.58 & 0.561 \\
\hline $\begin{array}{c}\text { AA \# } \\
\text { Aspirin }\end{array}$ & -.052 & 0.606 & -0.09 & 0.928 & 0.25 & 0.802 & -35.2 & - & 0.25 & 0.802 \\
\hline
\end{tabular}


Supplementary Information

Supplemental Material

This article is protected by copyright. All rights reserved 